\title{
How Do Tumor-Treating Fields Work?
}

\author{
Kristen W. Carlson, Jack A. Tuszynski, Socrates Dokos, Nirmal Paudel, \\ Thomas Dreeben, and Ze'ev Bomzon
}

\section{Introduction}

Since approved by the FDA for the treatment of glioblastoma brain cancer in 2015 , tumor-treating fields (TTFields) have rapidly become the fourth modality to treat cancer, along with surgery, chemotherapy, and radiation [1]. TTFields are now in clinical trials for a variety of cancer types. While efficacy has been proven in the clinic, higher efficacy is demonstrated in vitro and in animal models, which indicates much greater clinical efficacy is possible. To attain the great promise of TTFields, uncovering the mechanisms of action (MoA) is necessary.

TTFields are $200 \mathrm{kHz}$ AC electric fields directed transcranially or transdermally to a tumor site with target field strength of $2-4 \mathrm{~V} / \mathrm{cm}$. Through unknown MoA, TTFields kill cancer cells, extending survival for victims of brain cancer. Empirical studies show TTFields exert a variety of effects on cell processes [2-5]. And while the cause-effect chain is under investigation, they ultimately disrupt mitosis, the

K. W. Carlson $(\triangle)$

Department of Neurosurgery, Beth Israel Deaconess Medical Center/Harvard Medical School, Boston, MA, USA

e-mail: kwcarlso@bidmc.harvard.edu

J. A. Tuszynski

Department of Physics, University of Alberta, Edmonton, AB, Canada

S. Dokos

Department of Biomedical Engineering, University of New South Wales, Sydney, Australia

N. Paudel

Independent Consultant, Greenville, NC, USA

T. Dreeben

OSRAM Opto Semiconductors, Exeter, NH, USA

Z. Bomzon

Novocure Ltd., Haifa, Israel

(C) The Author(s) 2021

S. N. Makarov et al. (eds.), Brain and Human Body Modeling 2020,

https://doi.org/10.1007/978-3-030-45623-8_2 
delicately orchestrated process of cell division, which occurs more often in cancer cells than in healthy cells. This article will review the clues and hypotheses about TTFields MoA that have been uncovered to date. We present the novel hypothesis that the intrinsic, mitochondrial apoptotic pathway upregulated by Bcl-2 and inhibited by BAX is a key component of TTFields MoA.

\subsection{TTFields Affect Large, Polar Molecules}

Early hypotheses on TTFields MoA pointed to disruptive effects on subcellular structures such as individual dipoles, e.g., tubulin, which are common in the cell $[2,3]$. However, detailed subsequent calculations show that TTFields' effects on individual dipoles seem insufficient to disrupt their function (Fig. 1) [6]. Using parameters at the high end of their ranges, Tuszynski et al. calculated that the force on a free, unpolymerized 3000 debye tubulin dimer by $1 \mathrm{~V} / \mathrm{cm}$ TTFields is $10^{-24} \mathrm{~J}$, several orders of magnitude less than the cell's background thermal energy, and therefore too low to torque it (Table 1). On the other hand, larger molecules composed of many dipoles, such as microtubules (MT), septin, and organelles, may accumulate enough dipole moment in their structure to be significantly affected.

Further, it is likely that TTFields' energy is amplified at various locations within the cell. Identifying those amplifying locations is an important part of our research program [7].

TTFields could also modulate ion channel gates in the mitochondrial membranes, which in turn regulate cellular processes such as apoptosis (programmed cell death).
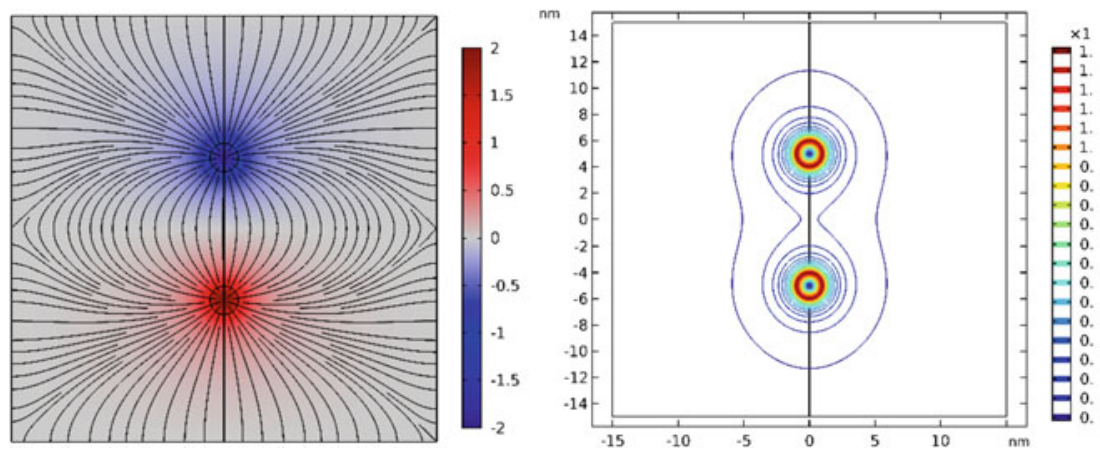

Fig. 1 COMSOL (Burlington, MA, USA) 2D axisymmetric finite element model of a dipole approximating a tubulin dimer. Left: color scale, electric potential in volts. Streamlines: electric field. Right: electric field norm contours in V/m; cf. to TTFields imposed field strength of $2 \times 10^{2} \mathrm{~V} / \mathrm{m}$. Model bears out calculations by Tuszynski et al. predicting the TTFields' direct effect on comparable subcellular dipoles is orders of magnitude too weak to have a disruptive effect on cell structures [6]. Thus, workers look for locations in the cell where TTFields' effect is amplified, such as in highly conductive microtubules composed of tubulin dimers, or at the cell furrow during late mitosis [7] 
Table 1 Three ubiquitous cellular energy levels, which, if exceeded via an exogenous source, would disrupt processes

\begin{tabular}{l|l}
\hline Disruption metric & Energy \\
\hline Background thermal energy & $k T=4 \times 10^{-21}$ joules \\
\hline Energy of ions crossing cell membrane (e.g. $\mathrm{K}+, \mathrm{Na}+)$ & $20-100 \mathrm{meV}$ \\
& $\sim 1-4 \mathrm{kT}$ \\
\hline Adenosine Triphosphate (ATP) & $\sim 25 k T$ \\
& $\approx 10^{-19}$ joules \\
\hline
\end{tabular}

Table 2 Signal-to-noise ratio guidance in signal theory

\begin{tabular}{l|l}
\hline Signal/noise $(\mathrm{dB})$ & Quality \\
\hline $1-10$ & Unreliable \\
\hline 20 & Intelligible but noisy \\
\hline 60 & High fidelity \\
\hline
\end{tabular}

Scale is logarithmic: $1 \mathrm{~dB}=10 \log _{10}\left(P_{1} / P_{2}\right)$ where $P$ is power (watts)

In general, cellular processes evolved to send signals in the presence of background thermal noise, whose value is given by Boltzmann's constant $k$ times absolute temperature $T$ [6]. For practical purposes, we assume energy required for disruption must be at least 1-2 orders of magnitude above $k T$, since the signal/noise ratio in a reliable system is never 1:1 (Table 2) [8]. Another ubiquitous energy threshold in cellular activities, for instance, in MT depolymerization or motor protein transport, is ATP energy (Table 1). Since ATP energy is the actual energy used by the cell for numerous binding or unbinding processes, and not a probabilistic floor as is $k T$, TTFields energy need not substantially exceed it to interfere with an ATP-driven process. While $k T$ and ATP energies are ubiquitous in the cell, other more specialized energy levels exist as well. Any cellular process in which TTFields' energy exceeds the threshold energy driving that process is a candidate for TTFields MoA.

Tuszynski et al. propose an "energetic constraint from above," $3 \times 10^{-12} \mathrm{~W}$, approximately the power generated by a cell, based on the hypothesis that TTFields cannot disrupt many normal and mitotic cellular processes, which would occur if the energy absorbed by the cell appreciably heated it [6], and empirically no significant heating effects have been observed [2]. However, while much of a cell's energy is devoted to heating it to a temperature where its biochemical reactions are possible, energy absorbed from TTFields may be concentrated at key structures and have nonthermal effects higher up on the entropic food chain.

\subsection{The Need for a "Complete" TTFields Theory}

We advocate constructing a "complete" theory of TTFields tying together the underlying mechanisms of TTFields' efficacy with its unobserved effects on 
Table 3 Ingredients of a complete TTFields theory

\begin{tabular}{l|l}
\hline Components of TTFields theory & Examples \\
\hline $\begin{array}{l}\text { Predicted electromagnetic effects on } \\
\text { subcellular structures }\end{array}$ & $\begin{array}{l}\text { Dielectrophoresis of large molecules near the cellular } \\
\text { furrow during late mitosis [9, 10] }\end{array}$ \\
\hline $\begin{array}{l}\text { Predicted electromagnetic effects on } \\
\text { cell-signaling pathways }\end{array}$ & $\begin{array}{l}\text { Triggering of intrinsic, Bcl2/BAX-mediated apoptosis by } \\
\text { direct effect on mitochondrial outer membrane (MOM) or } \\
\text { indirect effect via excess free tubulin obstructing MOM } \\
\text { voltage-gated ion channels }\end{array}$ \\
\hline $\begin{array}{l}\text { Observed effects of TTFields on } \\
\text { cellular structures }\end{array}$ & $\begin{array}{l}\text { Excess free tubulin [4] and decrease of septin at cell } \\
\text { midline [5], both correlated with mitotic spindle deformi- } \\
\text { ties and other mitotic aberrations, such as cell blebbing, } \\
\text { multiple nuclei, aneuploidy, and apoptosis in interphase }\end{array}$ \\
\hline $\begin{array}{l}\text { Verifiable predictions leading } \\
\text { toward maximum efficacy }\end{array}$ & $\begin{array}{l}\text { Apply direction changes from multiple directions so as to } \\
\text { increase the incidence of field aligned and orthogonal to } \\
\text { cell axis of randomly oriented cells in vivo } \\
\text { To treat edema in brain cancer, replace dexamethasone } \\
\text { (dex) with celecoxib to eliminate dex inhibiting TTFields' } \\
\text { triggering of intrinsic apoptosis [11] }\end{array}$ \\
\hline
\end{tabular}

subcellular structures, cell signaling pathways, and observed effects (Table 3). A complete theory will sort out what is causal versus epiphenomenal or downstream effects. For instance, it is unknown whether the decrease of the key motor protein septin at the cell midline [5] or the $25 \%$ increase in free tubulin in relative terms [4] in TTFields-treated cells are causing disruption of mitosis or are just side effects. Importantly, a complete theory imposes the maximum constraints on its formulation, which is helpful conceptually and in modeling TTFields effects. The several hypotheses on TTFields MoA (Sect. 3) conflict with each other and with empirical evidence in varying respects, which must be sorted out. Examining isolated TTFields phenomena results in under-constrained models.

\section{Empirical Clues to TTFields MoA}

In this section, we compile key clues informing and constraining a theory of how TTFields work.

\subsection{TTFields Only Kill Fast-Dividing Cells}

TTFields do not affect normal cells and only affect cells that divide more often than normal cells, i.e., cancer cells. Thus, MoA ideas focus on subcellular structures that differ in the cell division stages (mitosis) versus between cell division (interphase). For example, MTs become much more dynamic in their length during mitosis, and during mitosis some septin structures align with the cell axis, while other septin structures rotate orthogonal to it, while in interphase they are randomly aligned in general. 


\subsection{TTFields Require 2-4 V/cm Field Strength}

Figure 2 (left) shows a plot of killed versus live cells according to TTFields ambient field strength in vitro [2]. Based on this and other early works, numerous head and body modeling studies use $2 \mathrm{~V} / \mathrm{cm}$ as a threshold for efficacy required in TTFields. However, considering the weakened effect of TTFields when not aligned with the cell axis (Sect. 2.4) and the need to kill all cells in cancer to eliminate recurrence and metastases (tumor cells that spread to other organs from their original site), we suggest $4 \mathrm{~V} / \mathrm{cm}$ as an efficacy threshold.

\subsection{TTFields Are Frequency-Sensitive and Effective Only in the 100-300 $\mathrm{KHz}$ Range}

TTFields have proven effective at killing a variety of tumor cell types, but in all cases, the frequency range is confined to $100-300 \mathrm{KHz}$, equivalent to a period range of 3.3-10 $\mu$ s, and the specific frequency varies with tumor cell type (Fig. 2, right) $[2,3]$. For instance, TTFields' efficacy for glioblastoma and astrocytoma brain cancers, ovarian cancer, and mesothelioma lung cancer is maximal at $200 \mathrm{KHz}$, while for various types of lung, pancreatic, cervical, and mammary cancers, the peak effect is at $150 \mathrm{KHz}[4,12]$. We believe that part of the underlying mechanism is that the cell membrane becomes translucent to electromagnetic radiation in this
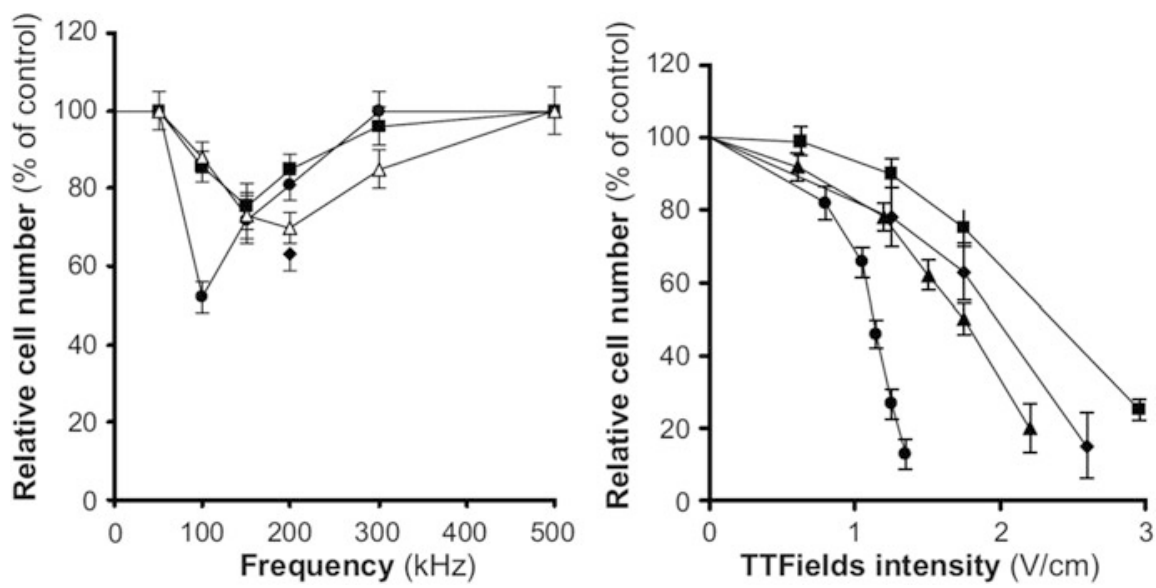

Fig. 2 Frequency- and electric field intensity-dependent TTFields' effects on proliferation of several cancer cell types. Left: relative change in number of cells after 24-h treatment of different frequencies at $1.75 \mathrm{~V} / \mathrm{cm}$. Right: effect of 24-h exposure to TTFields of increasing intensities (at optimal frequencies). B B16F1 (mouse melanoma). - MDA-MB-231 (human breast adenocarcinoma). $\boldsymbol{\Delta} \Delta$ F-98 (human glioma brain cancer). H1299 (small lung cell carcinoma). (From Kirson et al. [3], with permission, copyright (2007) National Academy of Sciences) 

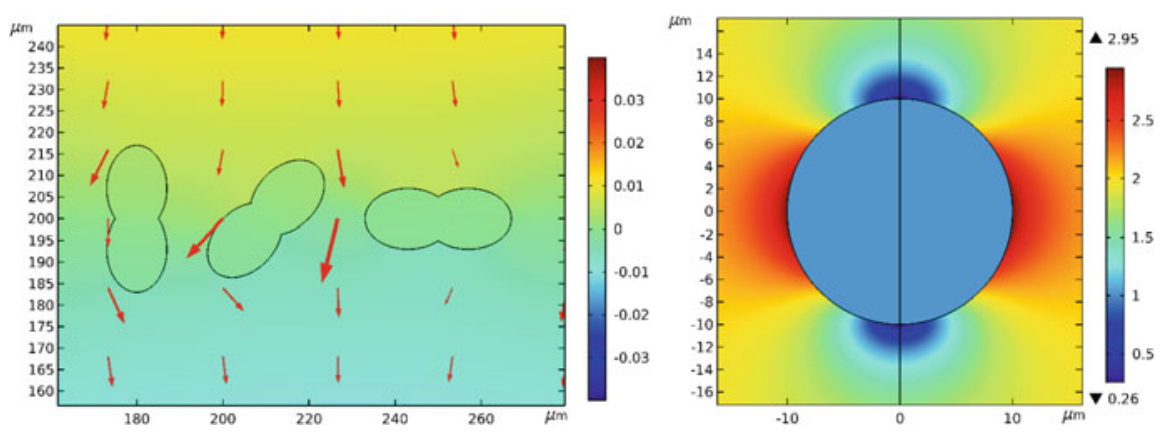

Fig. 3 At $200 \mathrm{KHz}$, a $10 \mu \mathrm{m}$ radius cell becomes "translucent" to an exogenous electric field (2 V/ $\mathrm{cm})$ due to complex permittivity of its membrane, while at lower frequencies it is opaque and at higher frequencies (e.g., MHz-GHz) it is transparent [9]. The field is largely, but not entirely shunted around the cell, with a fraction of its energy penetrating the cell to affect subcellular structures. Left: dividing cell at $0,45^{\circ}$, and $90^{\circ}$ (left to right) to the cell's axis. Electric potential (color bar, V) and current flow (arrows). Arrows: current density. Right: color bar: Electric field strength $(\mathrm{V} / \mathrm{cm})$

frequency range, allowing enough field strength to enter the cell and affect subcellular mitotic structures (Fig. 3) [9]. The mechanism underlying the upper limit of the range and different frequency responses is unknown, which could be, for instance, tied to relaxation time constants in DEP effects, the duration of sustained current flow at $\mathrm{MT}+$ end required to disrupt polymerization, or the duration required to charge the MT counter-ion/depleted ion layer/C-termini capacitor which in turn could disrupt MT functions.

The efficacy versus field strength relationship is nonlinear for all cell types (Fig. 2 right) and suggests a power law, for which there are at least two candidates. In general, energy and power in an electromagnetic wave are related to the square of field strength produced by the wave (Eq. 1). Dielectrophoretic force is proportional to the square of field strength (Eq. 2) [9]; see Zhao \& Zhang for a different analysis [13], and there are other possibly relevant power law relationships. It is notable that while the electric field strength squared is also proportional to frequency, TTFields efficacy does not increase with their frequency outside of the $100-300 \mathrm{KHz}$ range (Sect. 2.2). The underlying reason is unknown.

$$
\begin{gathered}
I_{\mathrm{ave}}=\frac{c \epsilon_{0} E_{0}^{2}}{2} \\
\left\langle\mathbf{F}_{\mathrm{DEP}}\right\rangle^{\sim} \nabla\left[\operatorname{Re}[\widetilde{\boldsymbol{E}}]^{2}+\operatorname{Im}[\widetilde{\boldsymbol{E}}]^{2}\right]
\end{gathered}
$$




\subsection{TTFields Are Highly Directional}

One in vitro experiment examined the proportion of cells killed in relation to the angle of the cell axis to the ambient TTFields direction [2]. This study found that TTFields are most lethal when aligned with the cell axis and have a weaker, secondary effect when orthogonal to the axis, but no significant effect at $45^{\circ}$ to the axis. We interpret these results to indicate there are at least two MoA, and they are not additive.

Following the conclusions of the experiments just described, and assuming cells are randomly oriented when they begin mitosis, it is logical to hypothesize that changing the direction of TTFields would subject more cells to field alignment or orthogonality than applying the treatment from one direction only. A possible tradeoff is the reduced duty cycle - the proportion of time spent in a given direction. It is possible that the field must be applied for a minimum duration in order to produce its disruptive effect on subcellular structures.

Thus, another study looked at TTFields' efficacy with no change of direction versus one change of direction twice per second [2]. The latter protocol killed 20\% more cells than TTFields applied with no change of direction.

A third set of experiments was published in US patent [14]. Figure 4, from the patent, compares the changes in growth rate of glioma F98 cells (brain cancer) subjected to $200 \mathrm{KHz}$ TTFields imposed from two orthogonal directions at different change-of-direction frequencies.

The several sets of experiments support each other, while the mechanisms underlying the increased tumor cell-killing effect with change-of-direction duty cycle remain to be understood.

\subsection{TTFields Have Their Strongest Effect in Prophase and Metaphase}

Kirson et al. also empirically examined TTFields efficacy at different points in the cell division cycle. They found that the strongest effects occur in metaphase, i.e., early in mitosis, with the field direction aligned with the cell axis [2]. Secondarily, when TTFields are orthogonal to the cell axis, they have a lesser effect, also in metaphase, specifically in prophase at the start of mitosis.

\subsection{TTFields Increase Free Tubulin and Decrease Polymerized Tubulin in the Mitotic Spindle Region}

Giladi et al., through careful experimentation, demonstrated that free tubulin is increased by $12.8 \%$ in absolute terms, which is $\sim 25 \%$ in relative terms, and 


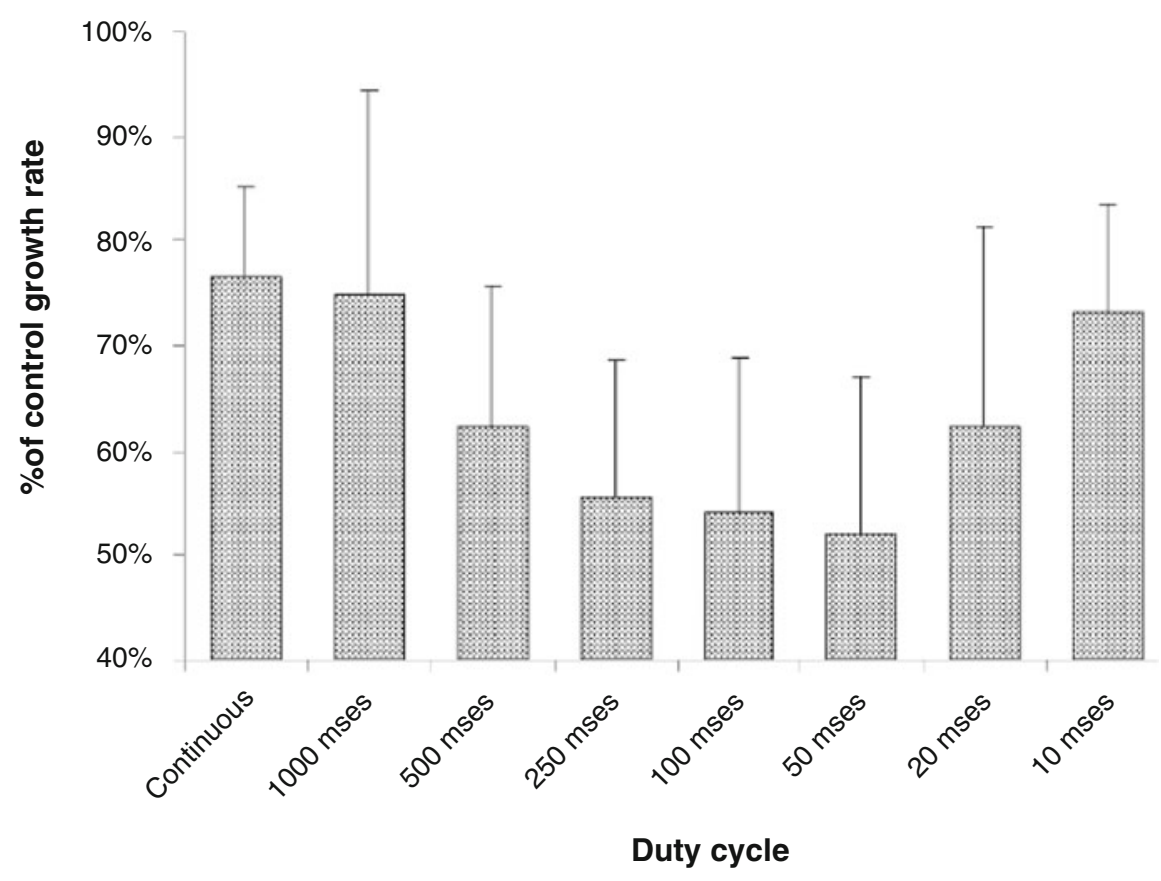

Fig. 4 (From Palti [14]). $200 \mathrm{KHz}$ TTFields were applied to brain cancer cells in culture from two orthogonal directions at different duty cycle frequencies (i.e., at different frequencies of change between the two directions). The greatest efficacy occurred when the changes of direction occurred every $50 \mathrm{~ms}$. The $50 \mathrm{~ms}$ optimal duty cycle may be a significant clue as to the underlying mechanism of action, for instance, a dielectrophoretic relaxation time in a large intracellular macromolecule

polymerized tubulin (i.e., in microtubules) is decreased concomitantly in TTFieldstreated cells [4]. The effect was similar to, but not as pronounced as, the increase of free versus polymerized tubulin due to a vinca-alkaloid chemotherapeutic agent, vinorelbine, and opposite to effects of the paclitaxel class of chemotherapeutic agents that stabilize MT polymerization, thereby decreasing the ratio of free versus polymerized tubulin. Giladi et al. also showed phenomena such as aberrant spindle formation, mitotic "slippage" (delayed mitotic phases), aneuploidy (abnormal number of chromosomes), and other effects that can trigger the intrinsic form of apoptosis or are emblematic of it.

These results raise the possibility that TTFields act like the vinca alkaloids to disrupt MT polymerization, but without the noted deleterious side effects of chemotherapy. In one study, TTFields were shown to be as effective as chemotherapy in treating brain cancer [15] and in others were shown to be synergistic with chemotherapy for lung cancer, decreasing the dosage required to achieve equal efficacy [16] and increasing efficacy when used in conjunction with several chemo agents [12]. 


\section{Candidate Mechanisms of Action (MoA)}

\subsection{Dielectrophoretic (DEP) Effects}

Several modeling studies have shown that as the mother and daughter cells elongate during late mitosis and enter cytokinesis (division into the two daughter cells), field strength is significantly amplified near the furrow joining the two cells [9, 10, 13]. Since DEP effects are proportional to the electric field amplitude squared (Eq. 2), DEP forces increase dramatically in late mitosis when cells are maximally elongated and field lines are most concentrated near the cell furrow. Further, it is easy to see that DEP effects are enhanced when TTFields are aligned with the cell axis, in accord with experimental data (Sect. 2.4).

However, DEP effects do not correspond to empirical data showing TTFields have no effect when oriented at $45^{\circ}$ to the cell axis and a moderate effect at $90^{\circ}$ (Sect. 2.4 and Fig. 5). Also arguing against the DEP MoA theory is their weak effect early in the mitotic cycle and strong effect in the late mitotic cycle, in contradiction to experimental data (Sect. 2.5).

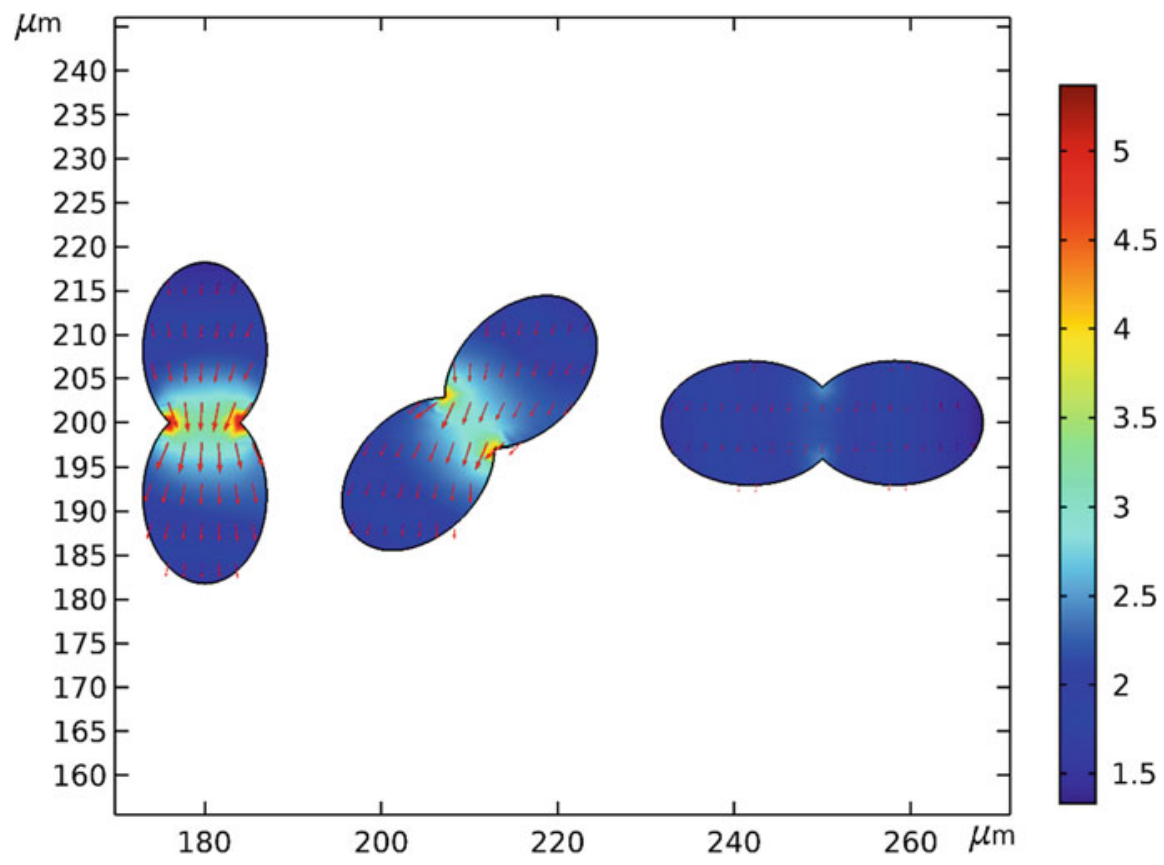

Fig. 5 A $10 \mu \mathrm{m}$ radius dividing cell in late mitosis approaching cytokinesis is shown with an exogenous $200 \mathrm{kHz}$ TTFields of $2 \mathrm{~V} / \mathrm{cm}$ imposed at $0,45^{\circ}$, and $90^{\circ}$ (left to right) to the cell's axis. Field strength (color bar, V/cm) and current flow (arrows) are amplified most when the field is aligned with the cell axis, less so at $45^{\circ}$, and not at all at $90^{\circ}$. (COMSOL model courtesy Wenger et al. [9]) 


\subsection{Microtubule Effects}

Several hypotheses have been proposed for TTFields disruption of MTs, including direct effects on C-termini state transitions, stall of the motor protein kinesin which transports cargo along the MT, and interference with MT polymerization. Since MTs become more active and increase in length and a proportion of them align with the cell axis as mitosis progresses, hypotheses that they are involved in TTFields MoA fit the constraints of acting on mitotic but not interphase cells and efficacy when the field is aligned or orthogonal to the cell axis (Sects. 2.1 and 2.4).

We calculate the force exerted by TTFields on elementary charges as follows. For each $100 \mathrm{~V} / \mathrm{m}=100 \mathrm{~N} / \mathrm{C}=100 \mathrm{~N} /\left(6.2415 \times 10^{18} \mathrm{e}\right)$, or $1.60218 \times 10^{-17} \mathrm{~N} / \mathrm{e}$ (where $\mathrm{N}$ is newtons and $\mathrm{e}$ is an elementary charge). For $8 \mathrm{C}$-termini charges and TTFields target strength of $200 \mathrm{~V} / \mathrm{m}$, this yields $2.56 \times 10^{-16} \mathrm{~N}$, orders of magnitude less than the minimum estimated $10^{-10}-10^{-12} \mathrm{~N}$ required to perturb $\mathrm{C}$-termini state transitions.

On the other hand, estimates vary for the stall force on the motor protein kinesin's "walk" along a MT, $8 \mathrm{~nm}$ dimer by dimer. One group separates each step of the walk into two phases: the first driven by the mechanical tension inherent in the kinesin "neck," which requires at least $\mathrm{pN}$ level force to stall, while the second, thermal energy-driven step of the walk which requires just $10^{-16} \mathrm{~N}$ to stall [17]. Thus, by the previous calculation and our modeling, disruption by TTFields of the kinesin walk is possible, and under the "complete" theory approach, attention is directed to correlating predicted effects of the disruption with what is observed.

Several possibilities exist for TTFields interference with MT polymerization. Direct action of TTFields on tubulin dimers at unamplified field strength, while suspected in early MoA proposals, is ruled out as described in Sect. 1.1. However, due to capacitive effects when TTFields are orthogonal to MTs, field strength may be amplified at the MT+ end where polymerization occurs. Second, Santelices et al. showed that MT conductivity is orders of magnitude greater than that of the ambient cytosol [18]. This quality alone implies that MTs will act as electrical shunts of TTFields-induced currents within the cell, which would have field amplification effects. Further, the conductive MT layer is likely the counter-ion layer attracted to the C-termini charges, and the mechanism of its conduction is uncertain [19]. If counter-ion resistance is non-Ohmic, e.g., not proportionate to MT length, possibly lossless, then as MT length increases in early mitosis, it would pick up an increasing field gradient, and if the resulting voltage, current, energy, or power exceeded a disruption threshold, e.g., for polymerization, that could explain why TTFields affect mitotic but not interphase cells (Sect. 2.1). This hypothesis is currently under investigation. 


\subsection{Septin Effects}

As mentioned, Gera et al. showed decreased septin concentration at the cell midline during mitosis, specifically as the cell enters anaphase, when errors in mitotic spindle formation become uncorrectable and ineluctably result in aberrant mitotic exit and/or programmed cell death [5]. Septin is, in fact, an ideal candidate for TTFields effects since septin family members self-assemble into various structures-hexameric and octameric quaternary structures, filaments, rings, and gauzes - that form rapidly and are precisely aligned or orthogonal to the cell axis during various mitotic phases, notably including prophase (Fig. 6; Sects. 2.4 and 2.5) [20-22]. Gera et al. estimate the dipole moment of the septin 2-6-7 complex at 2711 debye and note a higher value is possible when the complex is aggregated into higher-order septin structures.

They suggest that TTFields' effects on septin correlate with results showing that septin depletion leads to impairment of its cytoskeletal role to prevent improper

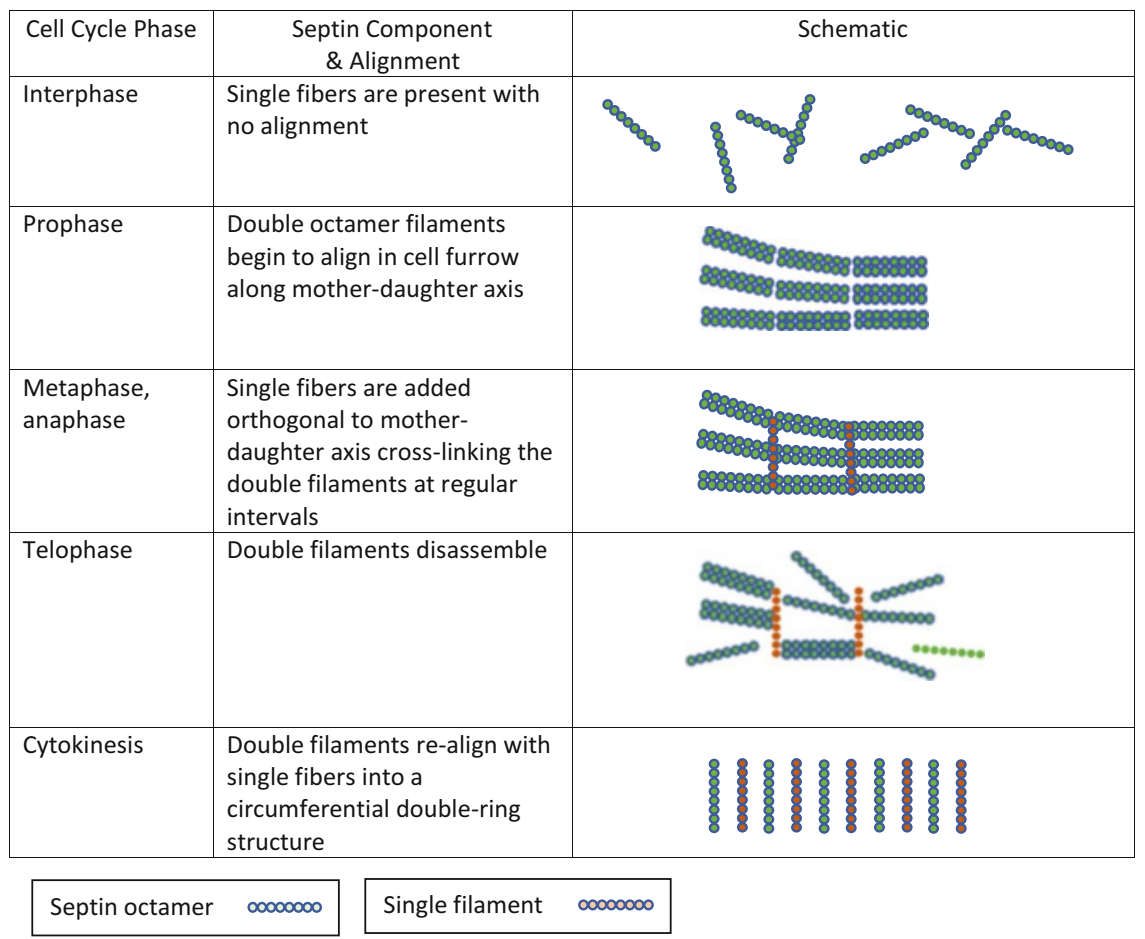

Fig. 6 Septin in the cell cycle (After Ong et al., [21]). For cell division to succeed, members of the cytoskeletal protein septin family are required to precisely assemble into various structures that are either aligned with or orthogonal to the cell axis. Some septins are large polarizable molecules, e.g., septin complex 2-6-7, which Gera et al. estimated its dipole moment at 2711 debye, and perhaps greater in higher-order structures [5]. Since TTFields can affect large polarized molecules and have their strongest effects when aligned or orthogonal to the cell axis, septins are a possible TTFields mechanism target. 
membrane shape, e.g., to stabilize aberrantly protruding membranes ("blebbing") against internal hydrostatic pressure as the cell changes shape drastically during later mitosis [20,23], and which they and others have observed in TTFields-affected cells [24]. Note, though, that Gilden et al. found that septin rapidly accumulates in conjunction with a second cytoskeletal protein, actin, at the site of the blebs, and so depletion of septin at the cell midline has no obvious connection with accumulation of septin at the cell membrane, while it could be tied to effects observed at the cell cleavage furrow [25]. Another possibility is that depletion of any septin at the midline could trigger septin depletion at other cell locations since septin depletion across family members is correlated, perhaps since septin complex formation is dependent on multiple septins [26]. Further, Gera et al. observed no septin depletion early in mitosis (cf. Sect. 2.4). In contrast, Estey et al. showed that depletion of septins 2 and 7 early in cytokinesis causes binucleation, which has been observed in TTFields-treated cells [26].

Gera et al. hypothesize that (1) TTFields torque the septin 2-6-7 complex and interfere with its self-assembly into a lattice as the cell enters anaphase; (2) anillin is then unable to recruit the septin complex to perform its role in preserving the structural integrity of the cell as it elongates toward cytokinesis, and (3) membrane blebbing triggers cell death via the immune system. While they speculate that p53-induced extrinsic, immune-driven apoptosis may be the ultimate cause of cell death and found evidence of $\mathrm{p} 53$-dependent $\mathrm{G}_{0 / 1}$ cell cycle block, they were unable to find greater expression of p53 in TTFields-treated cells. Further, p53 has a complex role in cell signaling, cell cycle checkpoints, and apoptosis [27], and non-p53-dependent apoptotic pathways are possible, as is p53 involvement in intrinsic, mitochondrial apoptosis versus extrinsic apoptosis (Sect. 3.4) $[28,29]$. While Gera et al. conclude that the role of p53 in TTFields MoA is unclear, their focus on septin and their results merit further investigation.

\subsection{Is Intrinsic Apoptosis the Key Signaling Pathway Triggered by TTFields?}

Wong et al. hypothesized that TTFields may induce an immune system response [24] and noted that the MoA of dexamethasone (dex), routinely prescribed to reduce inflammation and edema in brain cancer patients, is steroidal-driven reduction of immune system function [11]. They created two cohorts: one with dex dose reduced to $<4.1 \mathrm{mg} / \mathrm{day}$, and result was a significant extension of overall survival among the low-dex cohort, perhaps supporting the hypothesis that the immune system is involved in TTFields MoA.

An alternate hypothesis is possible. First, note that immune system activity was not implicated in the original in vitro studies where TTFields were shown to have higher efficacy than in human trials [2,3]. Second, immune response correlated with 


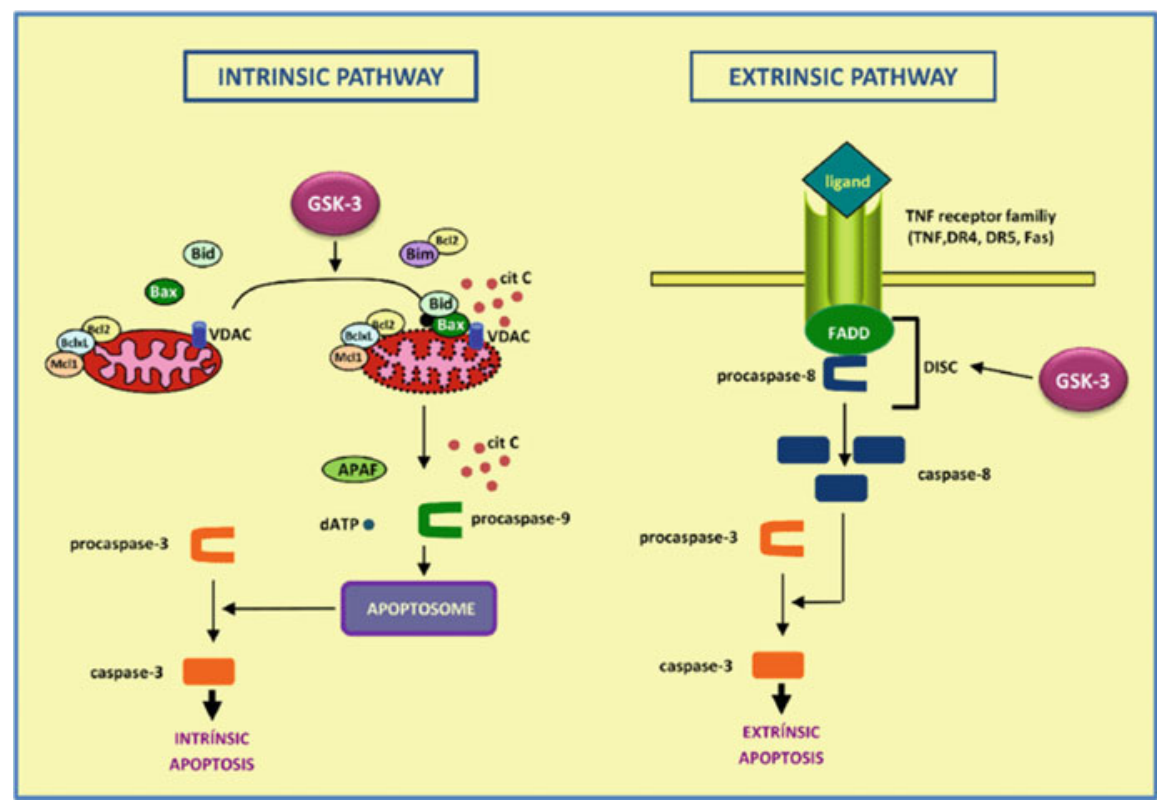

Fig. 7 Diagram showing the intrinsic, mitochondrial (left), and extrinsic, immune-related (right) apoptotic pathways. In the intrinsic pathway, the steroid dexamethasone (dex) is anti-apoptotic, while in the extrinsic pathway, dex is pro-apoptotic. Since dex interferes with TTFields efficacy, this suggests that intrinsic apoptosis is a key signaling pathway of its mechanism of action $(\mathrm{Mo} A)$. Since dex also interferes with radiotherapy and it is known that intrinsic apoptosis is a main MoA, it supports the intrinsic apoptosis theory of TTFields MoA and suggests that TTFields act like radiotherapy and vinca alkaloid chemotherapy, but notably without their deleterious side effects. (Source, with permission via ResearchGate: [39])

better response in human patients that occurred months into treatment [24], which again was not a factor in the in vitro efficacy occurring in a few days.

Third, in reviewing the literature on dex and apoptosis, we found that dex has pleiotropic effects on two different apoptotic pathways. In some tissues, dex enhances immune system, p53-driven ("extrinsic") apoptosis [30, 31], while in others, dex inhibits Bcl-2/BAX-regulated ("intrinsic" or "mitochondrial") apoptosis (Fig. 7) [32, 33]. Fourth, in fact, dex has been shown to inhibit intrinsic apoptosis in glioblastoma and is suspected to interfere with apoptosis in chemo- and radiation therapy [34-37]. Fifth, a principal MoA of radiotherapy, which induces DNA damage in target cells, is intrinsic apoptosis [38]. Thus, it seems likely that dex interferes with TTFields and radiotherapy for the same reason: inhibition of intrinsic apoptosis.

We hypothesize that TTFields trigger the intrinsic apoptotic pathway as a key component of its MoA and that the MoA of TTFields, vinca alkaloids, temozolomide, and radiotherapy overlap. It is notable that TTFields produce no side effects while chemo- and radiotherapy have serious deleterious effects on healthy, non-tumor cells and decrease quality of life for patients. 
The intrinsic apoptosis theory does not necessarily conflict with the hypotheses described above, which focus on electric field effects on subcellular structures. Rather, a complete TTFields theory requires tying field effects on subcellular targets to a downstream triggering of intrinsic apoptosis. One possibility is that TTFields have a direct or indirect effect on the mitochondrial outer membrane (MOM), which regulates intrinsic apoptosis via its ion channels. It has been shown that TTFields act on the ion channels directly, similar to electric field modulation of axon and neuron ion channels at much lower frequencies $(0-30 \mathrm{kHz})$ [40].

An indirect effect is also possible. Studies have shown that free tubulin has the correct conformation to block voltage-gated anion channels (VDAC) in MOM and that tubulin subtypes modulate MOM potential [41-43]. An excess concentration of free tubulin, as shown by Giladi et al. [4], could result in modulation of VDAC or other MOM ion channels and affect regulation of intrinsic apoptosis. Since tubulin regulates MOM channels via the degree to which subtypes block them [44, 45], this hypothesis can be tested by identifying the subtype(s) rendered free versus polymerized by TTFields and seeing if the same subtype(s) regulate the MOM channels that trigger intrinsic apoptosis.

Cells in which intrinsic apoptosis is triggered display similarities to the empirical observations of Giladi et al. of TTFields-treated cells, for instance, that many cells exit mitosis and die in interphase $[4,28]$.

The intrinsic apoptosis theory predicts that decreasing the Bcl-2/BAX ratio would enhance TTFields' efficacy as it does for radiotherapy and, concomitantly, increased Bcl-2/BAX ratio would explain TTFields resistance as it does for radiotherapy, notably in glioma cells [46-48].

Thus, the intrinsic apoptosis hypothesis has the appeal of possibly meeting the requirements of a "complete" TTFields theory (Sect. 1.2) tying together electromagnetic effects on intracellular structures, cell signaling pathways, the observed empirical effects of TTFields, and predictions leading to match clinical efficacy with the high in vitro and animal model in vivo efficacy.

\section{Conclusion}

Since 2004, a significant body of evidence has accumulated from which increasingly specific theories of how TTFields work have been proposed. Such theories must ultimately tie together the effects of electric fields on subcellular structures, cellsignaling pathways, empirically observed effects on cells, and predictions leading to clinical efficacy matching the high efficacy proven in vitro and in animal models. We have presented the leading candidates and highlighted the theory that TTFields trigger the intrinsic, mitochondrial-regulated, apoptotic pathway as a key component of their MoA. The intrinsic apoptosis theory suggests that TTFields' MoA is similar to that of vinca alkaloids and radiotherapy, but lacks their deleterious side effects, and predicts methods to enhance TTFields clinical efficacy. 
Acknowledgements Eduard Fedorov, Novocure Ltd., helped with dimensionless modeling. Magnus Olsson and Kiran Uppalapati, COMSOL, offered techniques for modeling on a nanometer scale. We thank Cornelia Wenger for sharing her COMSOL model. Jeffrey E. Arle, BIDMC/ Harvard Medical School, introduced the lead author to tumor-treating fields. Partial funding was provided by Novocure Ltd.

\section{References}

1. Mun, E. J., et al. (2017). Tumor-treating fields: A fourth modality in cancer treatment. Clinical Cancer Research, 24, 266-275.

2. Kirson, E. D., et al. (2004). Disruption of cancer cell replication by alternating electric fields. Cancer Research, 64, 3288-3295.

3. Kirson, E. D., et al. (2007). Alternating electric fields arrest cell proliferation in animal tumor models and human brain tumors. Proceedings of the National Academy of Sciences of the United States of America, 104, 10152-10157.

4. Giladi, M., et al. (2015). Mitotic spindle disruption by alternating electric fields leads to improper chromosome segregation and mitotic catastrophe in cancer cells. Scientific Reports, $5,18046$.

5. Gera, N., et al. (2015). Tumor treating fields perturb the localization of septins and cause aberrant mitotic exit. PLoS One, 10, e0125269.

6. Tuszynski, J. A., et al. (2016). An overview of sub-cellular mechanisms involved in the action of TTFields. International Journal of Environmental Research and Public Health, 13(11), 1128.

7. Tuszynski, J. A. (2019). The bioelectric circuitry of the cell. In S. Makarov, M. Horner, \& G. Noetscher (Eds.), Brain and human body modeling: Computational human modeling at EMBC 2018 (pp. 195-208). Cham: Springer. https://doi.org/10.1007/978-3-030-21293-3_11.

8. Pierce, J. R. (1981). Signals: The telephone and beyond. San Francisco: W.H. Freeman.

9. Wenger, C., et al. (2015). Modeling Tumor Treating Fields (TTFields) application in single cells during metaphase and telophase. Conference Proceedings: Annual International Conference of the IEEE Engineering in Medicine and Biology Society, 2015, 6892-6895.

10. Berkelmann, L., et al. (2019). Tumour-treating fields (TTFields): Investigations on the mechanism of action by electromagnetic exposure of cells in telophase/cytokinesis. Scientific Reports, 9, 7362.

11. Wong, E. T., et al. (2015). Dexamethasone exerts profound immunologic interference on treatment efficacy for recurrent glioblastoma. British Journal of Cancer, 113, 232-241.

12. Giladi, M., et al. (2014). Alternating electric fields (tumor-treating fields therapy) can improve chemotherapy treatment efficacy in non-small cell lung cancer both in vitro and in vivo. Seminars in Oncology, 41(Suppl 6), S35-S41.

13. Zhao, Y., \& Zhang, G. (2018). Elucidating the mechanism of $200 \mathrm{kHz}$ tumor treating fields with a modified DEP theory. IEEE International Symposium on Signal Processing and Information Technology (ISSPIT).

14. Palti, Y. (2011). Optimizing characteristics of an electric field to increase the Field's effect on proliferating cells. US patent 7,917,227 B2 US Application 11/537,026.

15. Stupp, R., et al. (2012). NovoTTF-100A versus physician's choice chemotherapy in recurrent glioblastoma: A randomised phase III trial of a novel treatment modality. European Journal of Cancer, 48, 2192-2202.

16. Kirson, E. D., et al. (2009). Chemotherapeutic treatment efficacy and sensitivity are increased by adjuvant alternating electric fields (TTFields). BMC Medical Physics, 9, 1.

17. Sozanski, K., et al. (2015). Small crowders slow down kinesin-1 stepping by hindering motor domain diffusion. Physical Review Letters, 115, 218102. 
18. Santelices, I. B., et al. (2017). Response to alternating electric fields of tubulin dimers and microtubule ensembles in electrolytic solutions. Scientific Reports, 7, 9594.

19. Priel, A., et al. (2005). Transitions in microtubule C-termini conformations as a possible dendritic signaling phenomenon. European Biophysics Journal, 35, 40-52.

20. Gilden, J. K., et al. (2012). The septin cytoskeleton facilitates membrane retraction during motility and blebbing. The Journal of Cell Biology, 196, 103-114.

21. Ong, K., et al. (2014). Architecture and dynamic remodelling of the septin cytoskeleton during the cell cycle. Nature Communications, 5, 5698.

22. Ong, K., et al. (2016). Visualization of in vivo septin ultrastructures by platinum replica electron microscopy. Methods in Cell Biology, 136, 73-97.

23. Stewart, M. P., et al. (2011). Hydrostatic pressure and the actomyosin cortex drive mitotic cell rounding. Nature, 469, 226-230.

24. Swanson, K. D., et al. (2016). An overview of alternating electric fields therapy (NovoTTF therapy) for the treatment of malignant glioma. Current Neurology and Neuroscience Reports, 16,8 .

25. Normand, G., \& King, R. W. (2010). Understanding cytokinesis failure. Advances in Experimental Medicine and Biology, 676, 27-55.

26. Estey, M. P., et al. (2010). Distinct roles of septins in cytokinesis: SEPT9 mediates midbody abscission. The Journal of Cell Biology, 191, 741-749.

27. Ozaki, T., \& Nakagawara, A. (2011). Role of p53 in cell death and human cancers. Cancers (Basel), 3, 994-1013.

28. Varmark, H., et al. (2009). DNA damage-induced cell death is enhanced by progression through mitosis. Cell Cycle, 8, 2951-2963.

29. Luna-Vargas, M. P., \& Chipuk, J. E. (2016). The deadly landscape of pro-apoptotic BCL-2 proteins in the outer mitochondrial membrane. The FEBS Journal, 283, 2676-2689.

30. Price, L. C., et al. (2015). Dexamethasone induces apoptosis in pulmonary arterial smooth muscle cells. Respiratory Research, 16, 114.

31. Li, H., et al. (2012). Glucocorticoid receptor and sequential P53 activation by dexamethasone mediates apoptosis and cell cycle arrest of osteoblastic MC3T3-E1 cells. PLoS One, 7, e37030.

32. Tsai, H. C., et al. (2015). Dexamethasone inhibits brain apoptosis in mice with eosinophilic meningitis caused by Angiostrongylus cantonensis infection. Parasites \& Vectors, 8, 200.

33. Lee, I. N., et al. (2015). Dexamethasone reduces brain cell apoptosis and inhibits inflammatory response in rats with intracerebral hemorrhage. Journal of Neuroscience Research, 93, $178-188$.

34. Das, A., et al. (2004). Dexamethasone protected human glioblastoma U87MG cells from temozolomide induced apoptosis by maintaining $\mathrm{Bax}: \mathrm{Bcl}-2$ ratio and preventing proteolytic activities. Molecular Cancer, 3, 36.

35. Das, A., et al. (2008). Modulatory effects of acetazolomide and dexamethasone on temozolomide-mediated apoptosis in human glioblastoma T98G and U87MG cells. Cancer Investigation, 26, 352-358.

36. Sur, P., et al. (2005). Dexamethasone decreases temozolomide-induced apoptosis in human glioblastoma T98G cells. Glia, 50, 160-167.

37. Pitter, K. L., et al. (2016). Corticosteroids compromise survival in glioblastoma. Brain, 139, $1458-1471$.

38. Rupnow, B. A., et al. (1998). Direct evidence that apoptosis enhances tumor responses to fractionated radiotherapy. Cancer Research, 58, 1779-1784.

39. Gomez-Sintes, R., et al. (2011). GSK-3 mouse models to study neuronal apoptosis and neurodegeneration. Frontiers in Molecular Neuroscience, 4, 45.

40. Neuhaus, E., et al. (2019). Alternating electric fields (TTFields) activate Cav1.2 channels in human glioblastoma cells. Cancers (Basel), 11, 110.

41. Rostovtseva, T. K., \& Bezrukov, S. M. (2012). VDAC inhibition by tubulin and its physiological implications. Biochimica et Biophysica Acta, 1818, 1526-1535. 
42. Maldonado, E. N., et al. (2010). Free tubulin modulates mitochondrial membrane potential in cancer cells. Cancer Research, 70, 10192-10201.

43. Carre, M., et al. (2002). Tubulin is an inherent component of mitochondrial membranes that interacts with the voltage-dependent anion channel. The Journal of Biological Chemistry, 277, 33664-33669.

44. Sheldon, K. L., et al. (2015). Tubulin tail sequences and post-translational modifications regulate closure of mitochondrial voltage-dependent anion channel (VDAC). The Journal of Biological Chemistry, 290, 26784-26789.

45. Rostovtseva, T. K., et al. (2018). Sequence diversity of tubulin isotypess in regulation of the mitochondrial voltage-dependent anion channel. The Journal of Biological Chemistry, 293, 10949-10962.

46. Azimian, H., et al. (2018). Bax/Bcl-2 expression ratio in prediction of response to breast cancer radiotherapy. Iranian Journal of Basic Medical Sciences, 21, 325-332.

47. Sun, D., et al. (2018). MicroRNA-153-3p enhances cell radiosensitivity by targeting BCL2 in human glioma. Biological Research, 51, 56.

48. Liu, J. J., et al. (2005). Expression of survivin and bax/bcl-2 in peroxisome proliferator activated receptor-gamma ligands induces apoptosis on human myeloid leukemia cells in vitro. Annals of Oncology, 16, 455-459.

Open Access This chapter is licensed under the terms of the Creative Commons Attribution 4.0 International License (http://creativecommons.org/licenses/by/4.0/), which permits use, sharing, adaptation, distribution and reproduction in any medium or format, as long as you give appropriate credit to the original author(s) and the source, provide a link to the Creative Commons license and indicate if changes were made.

The images or other third party material in this chapter are included in the chapter's Creative Commons license, unless indicated otherwise in a credit line to the material. If material is not included in the chapter's Creative Commons license and your intended use is not permitted by statutory regulation or exceeds the permitted use, you will need to obtain permission directly from the copyright holder.

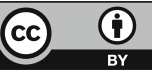

